\title{
Long-Lived Coherences for Homogeneous Line Narrowing in Spectroscopy
}

\author{
Riddhiman Sarkar, ${ }^{1}$ Puneet Ahuja, ${ }^{1}$ Paul R. Vasos, ${ }^{1, *}$ and Geoffrey Bodenhausen ${ }^{1,2}$ \\ ${ }^{1}$ Institut des Sciences et Ingénierie Chimiques, Ecole Polytechnique Fédérale de Lausanne, EPFL, Batochime, \\ 1015 Lausanne, Switzerland \\ ${ }^{2}$ Département de Chimie, associé au CNRS, Ecole Normale Supérieure, 24 Rue Lhomond, 75231, Paris Cedex 05, France
}

(Received 24 August 2009; published 2 February 2010)

\begin{abstract}
Line broadening, which can arise from inhomogeneities or homogeneous relaxation effects that lead to finite lifetimes of quantum states, is the Achilles' heel of many forms of spectroscopy. We show that line broadening may be considerably reduced by exploiting long lifetimes associated with superpositions of quantum states with different symmetry, termed long-lived coherences. In proton NMR of arbitrary molecules (including proteins) in isotropic solution, the slow oscillatory decays of long-lived coherences can yield spectra with very high resolution. This improvement opens the way to high-field magnetic resonance of molecular assemblies that are almost an order of magnitude larger than could be hitherto studied. Coherences between states of different symmetry may be useful in other forms of spectroscopy to cancel unwanted line broadening effects.
\end{abstract}

DOI: 10.1103/PhysRevLett.104.053001

PACS numbers: 33.25.+k, 61.05.Qr, 87.64.K-

Ever since the first atomic absorption spectra were observed in the 19th century, it has become obvious that the widths of spectral lines were broadened by phenomena such as Doppler effects in gases and molecular beams, Hubble redshifts in spectra from remote galaxies, crystal strains and packing defects in solids, random orientations of crystallites in powders, temperature gradients, variations in chemical composition, and inhomogeneous electric or magnetic fields.

In nuclear magnetic resonance spectroscopy (NMR), a perturbation by an oscillating field $B_{1}$ applied to spin states quantized in the main $B_{0}$ field can induce coherent superpositions of these states. Decoherence due to internal interactions (homogeneous relaxation) and to variations of the $B_{0}$ field (inhomogeneous broadening) limit the spectral resolution. The larger the size of the molecules or supramolecular assemblies, the slower the rotational diffusion, the faster the transverse relaxation, and the broader the spectral lines.

In pairs of coupled spins $I$ and $S$ that are partly isolated from the environment, both longitudinal and transverse relaxation are due predominantly to their mutual dipolar interaction. It has been discovered recently $[1,2]$ that longlived states (LLS), i.e., states with populations that have lifetimes $T_{\mathrm{LLS}}$ that are much longer than the longitudinal ("spin lattice") relaxation time $T_{1}$, may be obtained by creating spin order that is immune to dipolar relaxation. This can be achieved by creating a difference between the population of the singlet state $S_{0}$ and the mean population of the three triplet states (see below). In favorable cases, an improvement in the lifetime by a factor $T_{\mathrm{LLS}} / T_{1}=36$ could be obtained [3]. Spin polarization has been preserved for several minutes using LLS in nitrous oxide enriched in nitrogen-15 [4]. This has made it possible to study phenomena such as slow exchange or slow transport in dynamic equilibrium by NMR over much longer intervals.
Obtaining narrow lines requires exciting spin coherences with slow decay rates. Many approaches can contribute to narrowing spectral lines by reducing inhomogeneous broadening to reveal the underlying homogeneous widths, notably time reversal to form spin echoes [5,6]. Narrowing the underlying homogeneous linewidths is widely regarded as a far greater challenge. Homogeneous broadening of spectra in frequency domain reflects decoherence in time domain, which is driven by stochastic interactions.

In fortunate cases, some of these stochastic interactions may cancel each other. Thus, one may exploit the partial cancellation of the fluctuations of dipole-dipole (DD) interactions and anisotropic chemical shifts (CSA) if the relative orientations of the interaction tensors are favorable [7-11]. This effect has become popular under the acronym Transverse Relaxation Optimised SpectroscopY (TROSY) [12]. In methyl groups, similar interference effects between the dipolar interactions between protons can also lead to line narrowing [13]. These effects have brought the size of proteins and their complexes that are amenable to structural and dynamic studies by NMR from about $20 \mathrm{kDa}$ to ca. $50 \mathrm{kDa}$.

The present contribution demonstrates that the use of coherent superpositions between singlet and triplet states can lead to a significant resolution enhancement in spectroscopy. A transition between singlet and triplet states, which belong to different irreducible representations of the spin permutation group, is normally symmetry forbidden and therefore has a vanishing transition probability. Under certain circumstances, though, it turns out to be straightforward to excite coherent superpositions across these forbidden transitions, allow them to undergo a precession under the effect of the Hamiltonian, and convert them into observable signals after a variable evolution interval. We refer to these superpositions as long-lived coherences (LLC's). 
In contrast to the populations of long-lived states (LLS), which decay monoexponentially, the time dependence of LLC's is oscillatory and can be mapped point-by-point in the manner of two-dimensional (2D) spectroscopy. Although applications of 2D spectroscopy were initially confined to NMR, they now embrace many modern forms of spectroscopy $[14,15]$. The concept of LLC's may also be relevant to other forms of spectroscopy, provided the symmetry of the quantum states can be controlled by some experimental means. In our case, this is simply achieved by switching a radio-frequency (rf) field on or off: if the rf field is on, the states are symmetric or antisymmetric under a permutation of the spins; if it is switched off, the states have no permutation symmetry because different Larmor precession frequencies make the spins nonequivalent.

We show that the lifetimes of LLC's are significantly less prone to homogeneous broadening than conventional single-quantum coherences, and, in addition, that they are insensitive to inhomogenous broadening. Therefore, LLC lifetimes can be significantly longer than the transverse relaxation times $T_{2}$ of single-quantum coherences $(3<$ $T_{\mathrm{LLC}} / T_{2}<9$ ), depending on the correlation time of rotational diffusion. When the slow oscillatory decays of LLC's are monitored by two-dimensional spectroscopy, one obtains very narrow lines with half widths at half
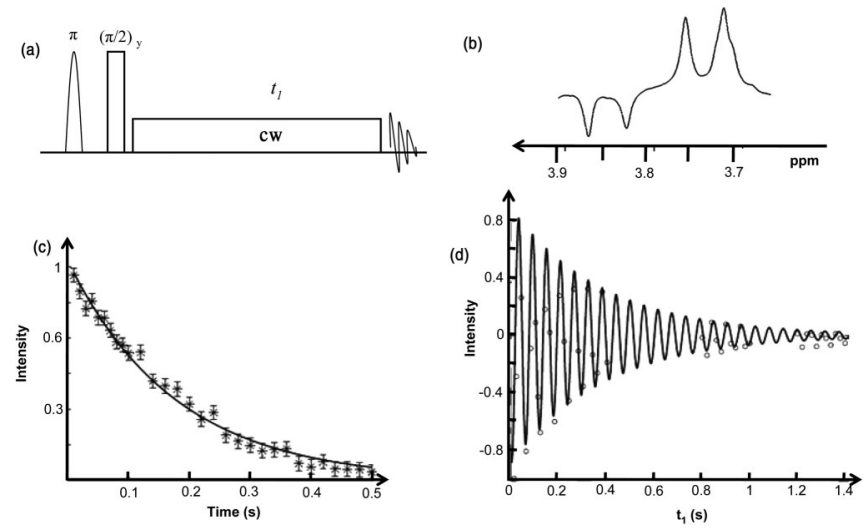

FIG. 1. Excitation and observation of long-lived coherences (LLC's) in high magnetic fields. (a) Pulse sequence for excitation of long-lived coherences. (b) Partial proton spectrum of the protein Ubiquitin $(2 \mathrm{mM}$ solution at $9.4 \mathrm{~T}$ or $400 \mathrm{MHz}$ and $300 \mathrm{~K}$ ) obtained with the pulse sequence in (a) with $t_{1}=120 \mathrm{~ms}$ and $\nu_{\mathrm{RF}}=\frac{1}{2}\left(\nu_{I}-\nu_{S}\right)$, where the only surviving signals are those of the protons $I=\mathrm{H}_{1}^{\alpha}$ and $S=\mathrm{H}_{2}^{\alpha}$ of Glycine-76 with $J_{I S}=-17.17 \mathrm{~Hz}$; (c) Experimental $T_{1 \rho}$ decay of ordinary inphase single-quantum coherences that are spin-locked by continuous-wave (cw) rf irradiation with the sequence but without the initial semiselective $\pi$ pulse, yielding the homogeneous relaxation rate $R_{1 \rho}=1 / T_{1 \rho} \approx R_{2}=1 / T_{2}=5.6 \mathrm{~s}^{-1}$, i.e., $T_{2}=180 \mathrm{~ms}$. (d) Oscillating signals (dots) due to the longlived coherence obtained using the full sequence (a). A fit to the function $I\left(t_{1}\right)=I_{0} \cos \left(2 \pi J_{I S} t_{1}\right) \exp \left(-R_{\mathrm{LLC}} t_{1}\right) \quad$ (solid line) yields $\left|J_{I S}\right|=17.17 \pm 0.08 \mathrm{~Hz}$ and $R_{\mathrm{LLC}}=2.5 \pm 0.7 \mathrm{~s}^{-1}$, i.e., $T_{\mathrm{LLC}}=400 \mathrm{~ms}$, so that $T_{\mathrm{LLC}} / T_{2}=2.2$. height $\Delta \nu_{\mathrm{LLC}}=\left(\pi T_{\mathrm{LLC}}\right)^{-1} \ll \Delta \nu=\left(\pi T_{2}\right)^{-1}$. In this Letter, we report the observation of linewidths $\Delta \nu_{\mathrm{LLC}}=$ $0.6 \mathrm{~Hz}$ in some amino acids in proteins. This corresponds to a line-narrowing effect by a factor $\sim 2.2$, since ordinary homogeneous linewidths of single-quantum coherences in the same systems are typically $\Delta \nu=1.4 \mathrm{~Hz}$. In principle, if external relaxation mechanisms can be silenced, a gain in linewidth by a factor $\sim 9$ should be possible in large macromolecules.

The singlet-triplet basis (see [16]) appropriate to describe a pair of equivalent spins with $I=\frac{1}{2}$ [3] comprises three symmetric triplet states and one antisymmetric singlet state:

$$
\begin{aligned}
& T_{+1}=|\alpha \alpha\rangle, \quad T_{0}=N(|\alpha \beta\rangle+|\beta \alpha\rangle), \\
& T_{-1}=|\beta \beta\rangle \quad \text { and } \quad S_{0}=N(|\alpha \beta\rangle-|\beta \alpha\rangle),
\end{aligned}
$$

where $N=2^{-1 / 2}$. In this work, we focus on long-lived coherences (LLC's) that correspond to off-diagonal elements of the density matrix [17-20]. A coherent superposition between the singlet and central triplet states can be readily defined in the singlet-triplet basis [3]:

$$
Q_{\mathrm{LLC}}=\left|S_{0}\right\rangle\left\langle T_{0}|+| T_{0}\right\rangle\left\langle S_{0}\right| .
$$

In the supplementary material, it is shown how this can be converted into Cartesian angular momentum operators that are more familiar in the field of NMR [21]:

$$
Q_{\mathrm{LLC}}=I_{x}-S_{x} .
$$

Because of the coherent effect of the scalar spin-spin interaction (see [16]) and relaxation, the LLC oscillates and decays:

$$
\frac{d}{d t} Q_{\mathrm{LLC}}=-\left(R_{\mathrm{LLC}}+i 2 \pi J_{I S}\right) Q_{\mathrm{LLC}} .
$$

The imaginary part leads to oscillations with a frequency that is determined by the scalar coupling constant $J_{I S}$, while the slow decay of the coherence is governed by the rate $R_{\mathrm{LLC}}$. Since the oscillation frequency of these coherences does not depend on the chemical shifts, and, by extension, on the local value of the $B_{0}$ field, they are insensitive to inhomogeneous broadening. As shown below, the lifetime $T_{\mathrm{LLC}}=1 / R_{\mathrm{LLC}}$ can be significantly longer than the transverse relaxation times $T_{2}=1 / R_{2}$ of the single-quantum coherences.

The coherent and relaxation properties of LLC's lead to 2D spectra with very high resolution, which can be used either to resolve nearly-degenerate signals or to measure $J$-couplings with an accuracy that is unprecedented in high field, albeit less accurately than in zero field [21]. In order to excite an LLC, one can readily transform the thermal equilibrium Boltzmann distribution $\left(I_{z}+S_{z}\right)$ into a difference $\left(I_{z}-S_{z}\right)$ by applying a semiselective $\pi$ pulse to one of the two doublets of the two-spin system (Fig. 1). Next, a $(\pi / 2)_{y}$ pulse is applied to excite $\left(I_{x}-S_{x}\right)$, followed by continuous-wave (cw) rf irradiation, which in effect sup- 
presses the chemical shifts, so that the system is best described in the singlet-triplet basis from there on. When the shifts are suppressed, the difference $\left(I_{x}-S_{x}\right)$ is converted into $Q_{\text {LLC }}$ (see [16]). The quantum yield of this conversion is $100 \%$.

The relaxation rate $R_{2}$ of single-quantum coherences in a system of two spins $I$ and $S$ is predominantly determined by the dipolar interaction between these spins [22]:

$$
R_{2}=\left(\frac{b_{I S}^{2}}{20}\right)[9 J(0)+15 J(\omega)+6 J(2 \omega)]+R_{2}^{\mathrm{ext}}
$$

where $b_{I S}=-\frac{\mu_{0}}{4 \pi} \frac{\gamma^{2} \hbar}{r_{I S}^{3}}$ is the strength of the homonuclear dipolar coupling, $r_{I S}$ the distance, $J(\omega)=\frac{\tau_{c}}{1+\omega^{2} \tau_{c}^{2}}$ the spectral density function (i.e., the Fourier transform of the autocorrelation function that describes the rotational diffusion of the molecule), $\tau_{c}$ the rotational correlation time, and the other quantities have their usual meaning. The term $R_{2}{ }^{\text {ext }}$ comprises contributions from external random fields, anisotropic chemical shifts (CSA), and dipole-dipole (DD) interactions to remote spins $K$.

In contrast to LLS, which are not affected by the dipolar interaction between the two spins $I$ and $S$, the relaxation rate constant $R_{\mathrm{LLC}}$ under this interaction is given by $[22,23]$ :

$$
R_{\mathrm{LLC}}=\left(\frac{b_{I S}{ }^{2}}{20}\right)[J(0)+3 J(\omega)+6 J(2 \omega)]+R_{\mathrm{LLC}}^{\mathrm{ext}}
$$

If the $R_{\mathrm{LLC}}{ }^{\text {ext }}$ term is small, the LLC has a longer lifetime than single-quantum coherences in all motional regimes, i.e., for arbitrary molecular tumbling rates. The ratio is $R_{\mathrm{LLC}} / R_{2}=1 / 3$ in the fast-tumbling limit $\left(\tau_{c} \ll 1 / \omega_{0}\right)$, when the spectral densities are equal at all frequencies, and $R_{\mathrm{LLC}} / R_{2}=1 / 9$ for slow tumbling $\left(\tau_{c} \gg 1 / \omega_{0}\right)$. Thus, if $R_{\mathrm{LLC}}{ }^{\mathrm{ext}}$ terms may be neglected, the ratio of the transverse relaxation times should increase from $T_{\mathrm{LLC}} / T_{2}=3$ in small molecules to $T_{\mathrm{LLC}} / T_{2}=9$ in macromolecules.

Experimentally, we have found that $T_{\mathrm{LLC}} / T_{2}=2.2$ in Gly-76 in Ubiquitin. In a small molecule (2,3,6trichlorophenol) in a field $B_{0}=9.4 \mathrm{~T}$ at room temperature, we have observed a ratio $T_{\mathrm{LLC}} / T_{2}=2$. The ratio $T_{\mathrm{LLC}} / T_{2}$ will be reduced by external contributions to the relaxation rates. On the other hand, the ratio $T_{\mathrm{LLC}} / T_{2}$ will not be degraded if the random fluctuations are symmetric with respect to spin permutation. This is analogous to the relaxation rates $R_{\mathrm{LLS}}$ of the populations of long-lived states, which are immune to fluctuations associated with CSA and DD tensors that are symmetrical under permutation of spins $I$ and $S[23,24]$.

The Fourier transform of LLC oscillations yields the magnitude of the $J_{I S}$ coupling constant with very high accuracy. The 2D spectrum shown in Fig. 2 reveals chemical shifts in the direct (horizontal) $\omega_{2}$ dimension, whereas the (vertical) $\omega_{1}$ dimension reveals the (aliased) scalar coupling constant $J_{I S}$. By choosing a coarse time incre-

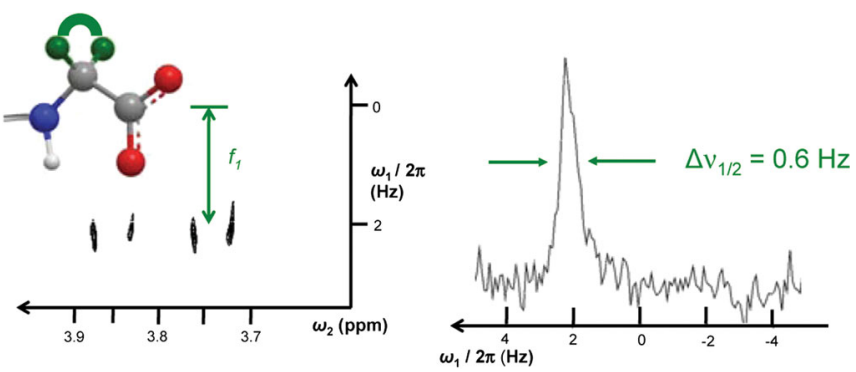

FIG. 2 (color). Observation of a long-lived coherence (LLC) in a two-dimensional (2D) proton spectrum of a large molecule with very narrow linewidths in high field (9.4 T). (Left) 2D spectrum of the LLC comprising the two inequivalent protons $\mathrm{H}_{1}^{\alpha}$ and $\mathrm{H}_{2}^{\alpha}$ (green spheres) of the terminal amino acid Gly-76 in the protein Ubiquitin (molecular mass $8.5 \mathrm{kDa}$, correlation time $\tau_{c}=4.5 \mathrm{~ns}$, at $300 \mathrm{~K}$ ). The spectral window was reduced to $10 \mathrm{~Hz}$ in the $\omega_{1}$ dimension (only $3 \mathrm{~Hz}$ shown), so that the folded peaks appear at $2.17 \mathrm{~Hz}$. (Right) Cross section extracted from the spectrum (a) at $\omega_{2}=3.84 \mathrm{ppm}$; the linewidth in the $\omega_{1}$ dimension is $0.6 \mathrm{~Hz}$.

ment $\Delta t_{1}$, the oscillations can be sampled up to $t_{1}{ }^{\max }=$ $N_{1} \Delta t_{1}$ to monitor the signal until it has almost completely decayed without requiring a large number of steps $N_{1}$. The 2D spectrum in Fig. 2 was recorded with $N_{1}=220$ points with increments $\Delta t_{1}=50 \mathrm{~ms}\left(t_{1}{ }^{\max }=11 \mathrm{~s}\right)$, so that the spectral window in the $\omega_{1}$ dimension was only $10 \mathrm{~Hz}$. Undersampling leads to spectral aliasing, but it is easy to correct for this effect and find that the signal appearing at $2.17 \mathrm{~Hz}$ corresponds in reality to $J_{I S}=-17.17 \mathrm{~Hz}$. The full linewidth at half-height of the signal is $\Delta \nu_{1 / 2}=$ $0.6 \mathrm{~Hz}$.

Recently, Pileio, Carravetta, and Levitt discussed similar transitions between symmetric and antisymmetric states in a different context. In their ingenious experiments [25], the Zeeman polarized sample was removed adiabatically from the magnetic field. It is then possible to bring about a Rabi nutation of the two-level system comprising the singlet and central triplet states by irradiation with an electromagnetic field at extremely low frequency (ELF), typically on the order of a few tens of $\mathrm{Hz}$ [25]. It has been shown that this rotation can lead either to the inversion of the populations across the singlet-triplet transition or to the creation of a singlet-triplet coherence that, like in our experiment, oscillates at the frequency of the $J$-coupling constant.

There are several differences between "ELF" and "LLC" experiments. In the former case, the Hamiltonian is switched adiabatically [25], in the course of a few seconds, by shuttling the sample outside the $B_{0}$ field. Therefore, longitudinal relaxation may take its toll, particularly for protons in biological macromolecules, as evidenced by field-cycling "relaxometry" experiments [26]. In ELF experiments, the desired coherence must then be excited by a slow Rabi nutation in zero field, which in practice requires an ELF pulse on the order of seconds to obtain a $\pi / 2$ nutation angle. By contrast, in our high-field LLC experiments, the spin system must be prepared by 
semiselective inversion of one or more chosen multiplets in the proton spectrum. This requires one or several rf pulses of $50 \mathrm{~ms}$ for a $\pi$ nutation angle, much shorter than $T_{1}$, although some losses due to $T_{2}$ relaxation during the pulses cannot be excluded. After semiselective inversion, a continuous rf field $B_{1}$ is suddenly switched on, superimposed on the high static $B_{0}$ field. This allows one to switch almost instantaneously (i.e., within a fraction of a $\mu$ s) to the desired Hamiltonian that is symmetrical under the spin permutation.

Like the popular TROSY method, the excitation of LLC's presupposes the existence of reasonably isolated scalar-coupled two-spin subsystems. Unlike TROSY however, no isotope labelling of nitrogen-15 or carbon-13 nuclei is required for LLC's, and the beneficial effects do not require high static magnetic fields. The isolation of subsystems can be improved through partial deuteration. This can be achieved by procedures that are well known for biological molecules [13].

LLC experiments are not limited to studying one pair of spins after another. The semiselective inversion of several spins in rapid succession (i.e., within an interval shorter than $T_{1}$ ) allows one to excite several LLC's simultaneously in different subsystems $I S, I^{\prime} S^{\prime}$, etc. In analogy to longlived states (LLS) [18,27-31], it should also be possible to excite and sustain LLC's in groups involving more than two coupled spins.

To summarize, long-lived coherences offer a means of obtaining homogeneous linewidths that are significantly narrower than in conventional spectroscopy. The enhancement in resolution may open the way to structural and dynamic NMR studies of macromolecules with a molecular mass that is almost an order of magnitude above the current limits. Measurements of relaxation rate constants $R_{\text {LLC }}$ can improve insight into dynamic processes. Generalizations to other forms of spectroscopy are conceivable if some means can be found to switch between symmetrical and nonsymmetrical Hamiltonians.

The authors thank Malcolm Levitt for numerous discussions. Martial Rey has provided valuable technical assistance. This work was supported by the Swiss National Science Foundation ("Ambizione" grant PZOOP2_121928 to P.V. and SNSF grant 200020_124694 to G.B. and P.V.), the Ecole Polytechnique Fédérale de Lausanne (EPFL), the Swiss Commission for Technology and Innovation (CTI grant 9991.1 PFIW-IW to G. B., Paul Dyson, Jean-Philippe Ansermet, and P. V.), and the French CNRS. P. V. thanks the Faculty of Basic Sciences, EPFL, for financial support.

*Corresponding author.

Paul.Vasos@epfl.ch

[1] M. Carravetta, O. G. Johannessen, and M. H. Levitt, Phys. Rev. Lett. 92, 153003 (2004).
[2] M. Carravetta and M. H. Levitt, J. Am. Chem. Soc. 126, 6228 (2004).

[3] R. Sarkar, P. R. Vasos, and G. Bodenhausen, J. Am. Chem. Soc. 129, 328 (2007).

[4] G. Pileio, M. Carravetta, E. Hughes, and M. H. Levitt, J. Am. Chem. Soc. 130, 12582 (2008).

[5] P. Pelupessy, E. Rennella, and G. Bodenhausen, Science 324, 1693 (2009).

[6] C. A. Meriles, D. Sakellariou, H. Heise, A. J. Moule, and A. Pines, Science 293, 82 (2001).

[7] M. Goldman, J. Magn. Reson. 60, 437 (1984).

[8] H. Shimizu, J. Chem. Phys. 40, 3357 (1964).

[9] E. L. Mackor and C. MacLean, J. Chem. Phys. 44, 64 (1966).

[10] S. Wimperis and G. Bodenhausen, Chem. Phys. Lett. 140, 41 (1987).

[11] S. Wimperis and G. Bodenhausen, Mol. Phys. 66, 897 (1989).

[12] K. Pervushin, R. Riek, G. Wider, and K. Wuthrich, Proc. Natl. Acad. Sci. U.S.A. 94, 12366 (1997).

[13] V. Tugarinov, P. M. Hwang, J. E. Ollerenshaw, and L. E. Kay, J. Am. Chem. Soc. 125, 10420 (2003).

[14] T. Brixner, J. Stenger, H. M. Vaswani, M. Cho, R. E. Blankenship, and G. R. Fleming, Nature (London) 434, 625 (2005).

[15] S. Mukamel, A. Piryatinski, and V. Chernyak, Acc. Chem. Res. 32, 145 (1999).

[16] See supplementary material at http://link.aps.org/ supplemental/10.1103/PhysRevLett.104.053001.

[17] G. Pileio and M.H. Levitt, J. Magn. Reson. 187, 141 (2007).

[18] P. Ahuja, R. Sarkar, P. R. Vasos, and G. Bodenhausen, Chem. Phys. Chem. 10, 2217 (2009).

[19] R. Sarkar, P. Ahuja, D. Moskau, P. R. Vasos, and G. Bodenhausen, Chem. Phys. Chem. 8, 2652 (2007).

[20] K. Gopalakrishnan and G. Bodenhausen, J. Magn. Reson. 182, 254 (2006).

[21] R. R. Ernst, G. Bodenhausen, and A. Wokaun, Principles of Nuclear Magnetic Resonance in One and Two Dimensions (Oxford University Press, Oxford, 1987).

[22] J. Cavanagh, W. J. Fairbrother, A. G. Palmer, M. Rance, and N. J. Skelton, Protein NMR Spectroscopy: Principles and Practice (Elsevier Science, San Diego, 2006), 2nd ed.

[23] P. Ahuja, R. Sarkar, P. R. Vasos, and G. Bodenhausen, J. Chem. Phys. 127, 134112 (2007).

[24] R. Sarkar, P. Ahuja, P. R. Vasos, and G. Bodenhausen, Chem. Phys. Chem. 9, 2414 (2008).

[25] G. Pileio, M. Carravetta, and M. H. Levitt, Phys. Rev. Lett. 103, 083002 (2009).

[26] I. Bertini, M. Fragai, C. Luchinat, and G. Parigi, Magn. Reson. Chem. 38, 543 (2000).

[27] G. Pileio, M. Concistré, M. Carravetta, and M. H. Levitt, J. Magn. Reson. 182, 353 (2006).

[28] R. Sarkar, D. Moskau, F. Ferrage, P. R. Vasos, and G. Bodenhausen, J. Magn. Reson. 193, 110 (2008).

[29] E. Vinogradov and A. K. Grant, J. Magn. Reson. 188, 176 (2007).

[30] D. Canet, S. Bouguet-Bonnet, C. Aroulanda, and F. Reineri, J. Am. Chem. Soc. 129, 1445 (2007).

[31] S. Cavadini and P. R. Vasos, Concepts Magn. Reson. A 32, 68 (2008). 